\title{
Internet-based hardware/software co-design framework for embedded 3D graphics applications
}

\author{
Chi-Tsai Yeh ${ }^{1,2^{*}}$, Chun-Hao Wang ${ }^{1}$, Ing-Jer Huang ${ }^{1}$ and Weng-Fai Wong ${ }^{3}$
}

\begin{abstract}
Advances in technology are making it possible to run three-dimensional (3D) graphics applications on embedded and handheld devices. In this article, we propose a hardware/software co-design environment for 3D graphics application development that includes the 3D graphics software, OpenGL ES application programming interface (API), device driver, and 3D graphics hardware simulators. We developed a 3D graphics system-on-a-chip (SoC) accelerator using transaction-level modeling (TLM). This gives software designers early access to the hardware even before it is ready. On the other hand, hardware designers also stand to gain from the more complex test benches made available in the software for verification. A unique aspect of our framework is that it allows hardware and software designers from geographically dispersed areas to cooperate and work on the same framework. Designs can be entered and executed from anywhere in the world without full access to the entire framework, which may include proprietary components. This results in controlled and secure transparency and reproducibility, granting leveled access to users of various roles.
\end{abstract}

Keywords: Hardware/software co-design, SystemC, Electronic system level, Internet, 3D graphics SoC, Heterogeneous hardware interface, Virtual machine

\section{Introduction}

3D graphics applications have gained significant popularity in recent decades. The market for both 3D graphics gaming applications and 3D video applications on mobile platforms is growing rapidly. The requirements as well as capabilities of consumer electronics such as personal digital assistants (PDAs), cell phones, global positioning systems (GPSs), and immersive teleconferencing vary significantly. In particular, screen sizes, resolutions, realtime and energy requirements differ. It is therefore challenging to meet such diversity all within certain design time.

System-level design has attracted much attention because of its ability to cope with the growing complexity of designs. Designing in this way raises the level of abstraction of the primary specification, allowing designers to explore the architectural trade-offs and hardware/software partition decisions that need to be made at a higher level. Nevertheless, the solution being

\footnotetext{
* Correspondence: yehchitsai@mail.kh.usc.edu.tw

'Department of Computer Science and Engineering, National Sun Yat-Sen University, Kaohsiung, Taiwan

Full list of author information is available at the end of the article
}

developed is usually just a part of a larger eco-system that consists of other hardware and software.

Electronic system-level (ESL) design and verification [1], shown in Figure 1, has been proposed to shorten the development time of embedded applications. The use of ESL can also help designers to meet some of the challenges mentioned previously.

The TLM interface standard [2] provides an essential ESL mechanism for architecture analysis, software development, software performance analysis, and hardware verification by separating computation and communication. Classifications of TLM are can be found in [3]. Pasricha et al. [4] demonstrated how the TLM approach can be used to model an SoC platform for architecture exploration.

System-level design languages (SLDLs) allow designers to represent of a system at multiple levels of abstraction. Two leading SLDLs, SystemC [5] and SpecC [6], support TLM using the channel concept. SystemC has garnered the most industry support in the United States, while SpecC lacks industry support [7]. SystemC is a modeling language built on top of standard $\mathrm{C}++$ by extending the language with class libraries. Kogel et al. [8] used SystemC in exploring the design space of a 3D graphics processor.

\section{SpringerOpen ${ }^{\circ}$}

(C) 2011 Yeh et al; licensee Springer. This is an Open Access article distributed under the terms of the Creative Commons Attribution License (http://creativecommons.org/licenses/by/2.0), which permits unrestricted use, distribution, and reproduction in any medium, provided the original work is properly cited. 


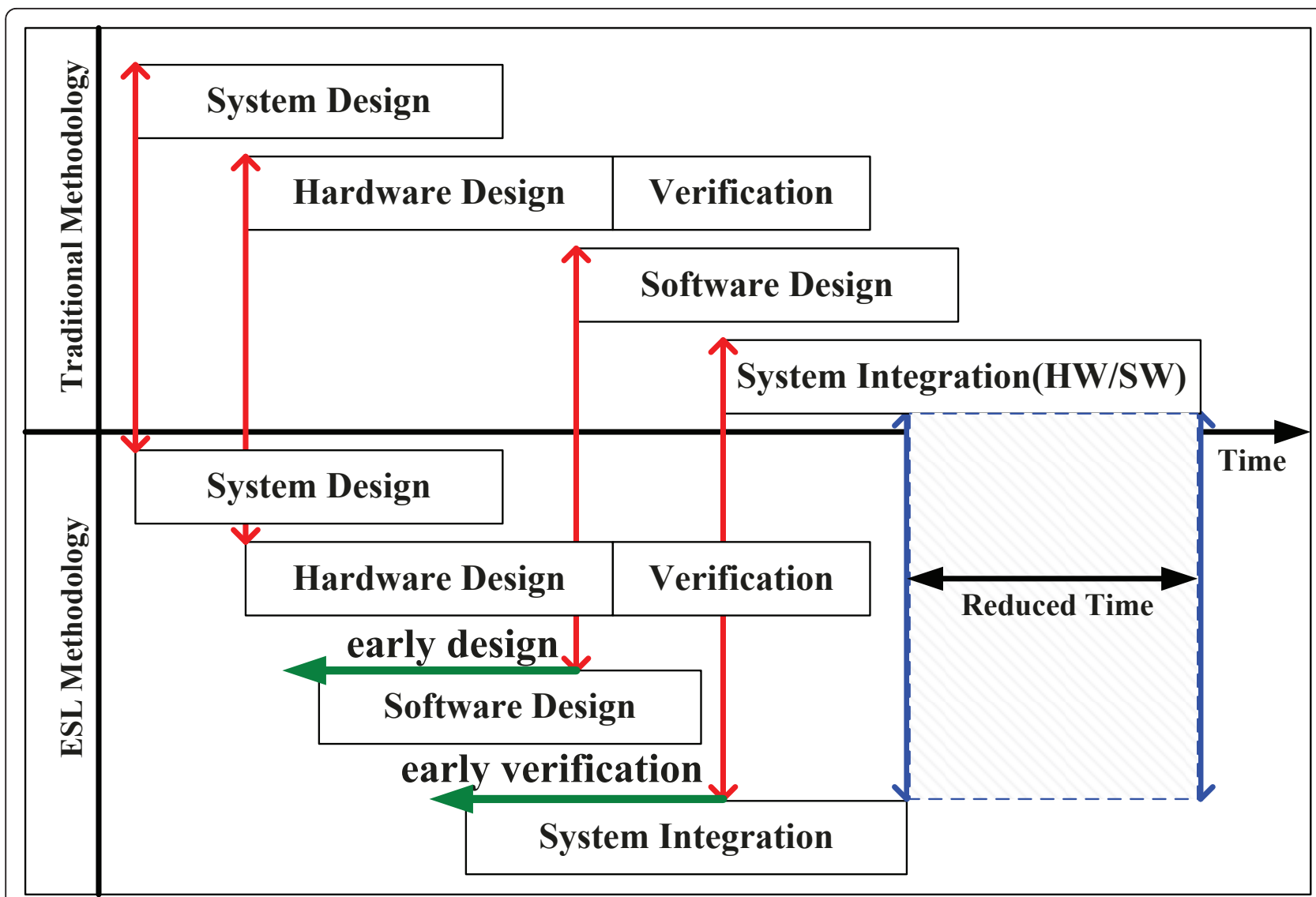

Figure 1 The comparison of traditional and ESL methodology.

They implemented a system architecture that could cope with the demands of $3 \mathrm{D}$ graphics processing and its internal memory bandwidth requirements. Crisu et al. $[9,10]$ presented a design exploration framework for an embedded 3D graphics accelerator called GRAAL. GRAAL is an open system that offers a coherent development methodology, based on an extensive library of SystemC/register transfer level (RTL) models of graphics pipeline components.

Rupp et al. [11] assume a design process structure consisting of an algorithmic, architectural, and implementation stage, as shown in the left-hand side of Figure 2. The design of embedded systems usually starts with the socalled algorithmic stage. Development at the algorithmic stage is aided by such electronic design automation (EDA) tools as Matlab/Simulink, CoWare SPW, and Synopsys CoCentric System Studio. Ram Rajagopal et al. [12] propose a rapid prototyping tool, which leads itself to a very smooth transition from design to implementation, allowing for powerful cosimulation strategies. Ptolemy [13] shows the importance of using higher-level representation constructs to build real-time functionality. Pelcat et al. [14] present an open-source Eclipse-based framework, which aims to facilitate the exploration and development processes in this context.

The availability of modeling architectural tools is inadequate when compared to the other two stages of the design process. The only familiar language at this stage is SystemC, introduced earlier. Synopsys ConvergenSC, which is part of Platform Architect [15], supplies many mature components using SystemC and provides a convenient environment in which integrates SystemC and RTL components.

The Galaxy Implementation Platform [16] is a comprehensive solution that supports the implementation stage, and is also referred to as the open Milkyway database [17]. This environment is capable of integrating a wide range of Synopsys commercial EDA tools and third-party EDA tools.

Figure 3 presents a recent survey of the most popular EDA tools. As the figure shows, there is no single EDA tool that supports the entire design process from initial concept to final product. The article provides a loose structure of hardware/software co-design. Hardware and software designers facilitate their familiar tools/methods on their own under the proposed environment presented in next section. 


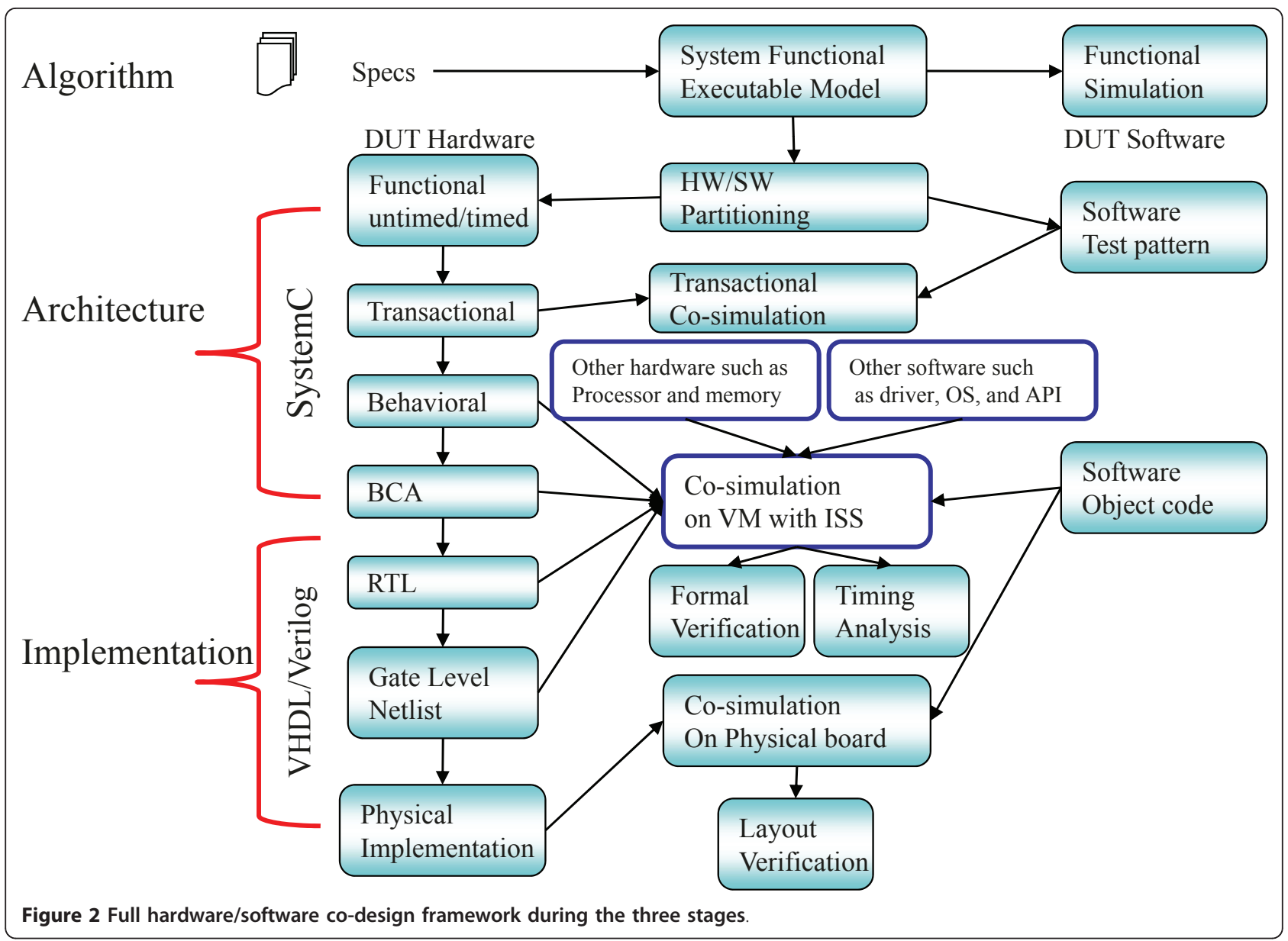

Previous hardware/software co-design frameworks have only considered the design-under-test (DUT) application for hardware components. However, a realistic system usually contains full hardware, consisting of processors, main memory, vector interrupt controller (VIC), and so on, and full software, such as device drivers, an operating system (OS), APIs, file system, and so on. Thus, additional hardware and software should be

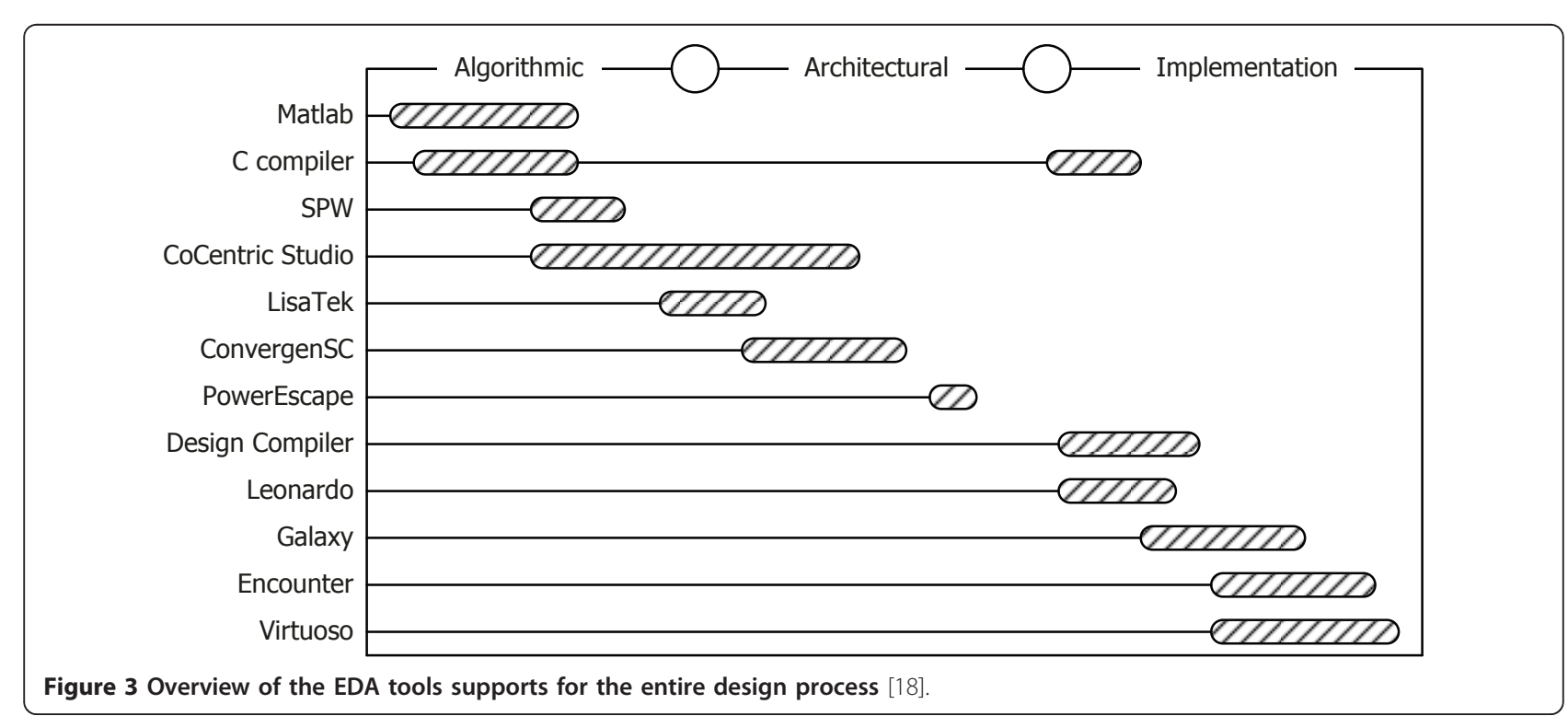


added for building the complete hardware/software cosimulation system, as shown in the middle of Figure 2.

Obviously, it exists a huge gap from a standalone hardware/software application to complete system environment. Therefore, the article proposes a framework for hardware/software co-design that permits multiple teams that are geographically dispersed to reproduce each others' results, and to cooperate in the architecture stage of the design by means of the Internet. This allows the software team to go into development at the earliest possible time.

The rest of the article is organized as follows. We will first describe the concept of our proposed framework and then describe the hardware and software aspects in detail. Followed by our experiment results and reproduces the proposed platform in different parts of the world. Finally, we conclude.

\section{Internet-based hardware/software co-design in the architecture stage}

Figure 2 outlines the hardware/software co-design framework. Then, Figure 4 explains in detail the Internetbased co-design method in the architecture stage. In the implementation development stage, a hardware vendor delivers the hard/soft intellectual property (IP) core(s) to the software team during their development of the system. Software designers may need to download a bin stream of an IP core into a field programmable gate array (FPGA), and then plug the FPGA into a development board, for example, Versatile Platform Baseboard (PB) [B19] and Leopard 6 SoC Design Platform [20], to start software development.

There are significant drawbacks to this process. First, the IPs have to be in place before the software team can start working. This can potentially lengthen the time to market, or corners such as the verification time will have to be cut. Secondly, the software team needs to learn how to use the EDA tools or the FPGA to work on the hardware. This adds new risk factors for verification, because the errors may be caused by the hardware design or simply malfunctions of the FPGA itself. Finally, there is an additional cost factor when using EDA tools or FPGA development platforms, especially if the software team does not already possess them.

In our proposed framework, software designers join the system development at the architecture stage, shown in Figure 2. However, because the development environment is the same for everyone involved, the software designers will consider that they co-design with the hardware team at the physical implementation (FPGA) stage.

QEMU [21] provides a full system simulation platform, which includes microprocessors, peripheral devices, memories, interconnection buses, etc., to act as a virtual machine (VM). It is also able to boot and run an unmodified commercial operating system like as Linux. Jing-
Wun Lin et al. [22] proposed a full system simulation that extended the QEMU-SystemC project provided by GreenSocs [23]. They presented a high-performance framework for hardware/software co-design and coverification. Unlike their framework, by leveraging virtualization, our proposal allows the software designers to run their program on QEMU, serviced by hardware IPs located in another part of the world. This allows the hardware and software teams to reproduce each other's work rapidly over the Internet. Using a TLM model and the standard protection provided by the Internet allows both sides to hide the content of their IPs, without compromising any functionality. Both teams can verify their own work using this framework.

Table 1 shows examples of the hardware and software components. We applied our framework to the design of a $3 \mathrm{D}$ graphics hardware accelerator that is integrated in a SoC platform. Using generic 3D graphics application on a workstation, the software team can produce the results they desire without the hardware or a device driver. This allows them to develop the upper layers of the software stack. However, in the lower layers, the device drivers cannot be written and tested without the hardware. Our framework enables the software and hardware teams to adopt a uniform set of test patterns for verifying their designs.

Reproducibility is a key aspect of our framework. It allows for instant feedback on the latest committed design, be it hardware or software, from other members of the team. Yet, the use of TLM means that access to the inner details of each component can be controlled, monitored, and managed. For example, if the hardware team do not wish to reveal an IP to the other members, they can do so by exposing only its interfaces, so that users can still use the component for their own subsystems. The use of the Internet does carry some risks, but there are already a large set of established protocols and mechanisms, such as the secure sockets layer (SSL) or virtual private network (VPN), that can minimize any risks caused.

\section{Software development environment}

From a software perspective, a full system consists of applications, the operating systems, device drivers, API, etc. In our 3D case study of hardware-software design flow, the software development environment is shown in Figure 5, and the factors of the corresponding components is shown in Table 2. We explain further the components in the sections below.

\section{Requisition for building guest software environment}

A strategic design choice we made from the start of the project was to use open source resources and industry standards. ARM processors are a common processor to embedded systems. The software designers build the 


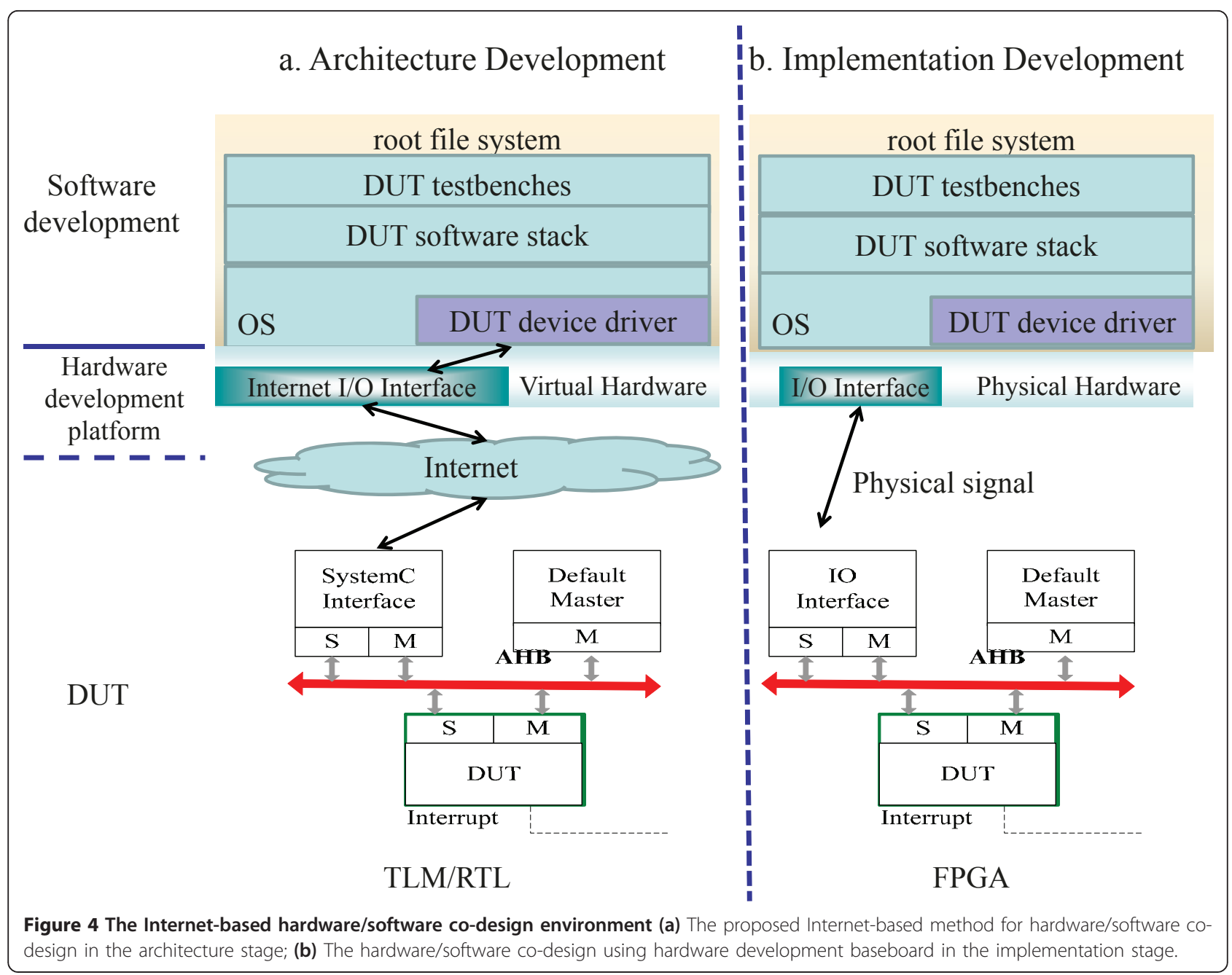

guest ARM software environment under host Intel x86 processor. They facilitate cross-platform compiler, GNU Compiler Collection (GCC) [24], to build the guest software, such as Linux kernel [25,26], Busybox [27], 3D graphics device driver, OpenGL ES API, 3D graphics test benches, and so on. Then, the software designers configure QEMU platform as Versatile PB and build QEMU as an executable program under host platform. Finally, they execute the software under guest platform QEMU.

\section{D Graphics Device Driver}

According to the $3 \mathrm{D}$ graphics test benches shown in Figure 6, The software designers implemented four functions in the device driver. When the user inserts the 3D graphics device driver into the kernel, the function 3D_init_module() allocates the necessary memory blocks, such as the $3 \mathrm{D}$ vertex buffer, $2 \mathrm{D}$ vertex buffer, $\mathrm{Z}$ buffer, and 32-bit temporary frame buffer. It also informs the Geometry Engine (GE) and the Rendering

Table 1 The examples of hardware/software co-design components

\begin{tabular}{cc}
\hline HW/SW Co-design components & Examples \\
\hline Software development & OS, file system, device driver, API, test benches, and so on. \\
\hline Hardware development platform & QEMU, VirtualBox, etc. \\
\hline Design Under Test (DUT) & Versatile PB development baseboard, Leopard 6 SoC Design Platform, etc. \\
\cline { 2 - 2 } & PhystemC - Synopsys EDA tool), \\
\hline
\end{tabular}




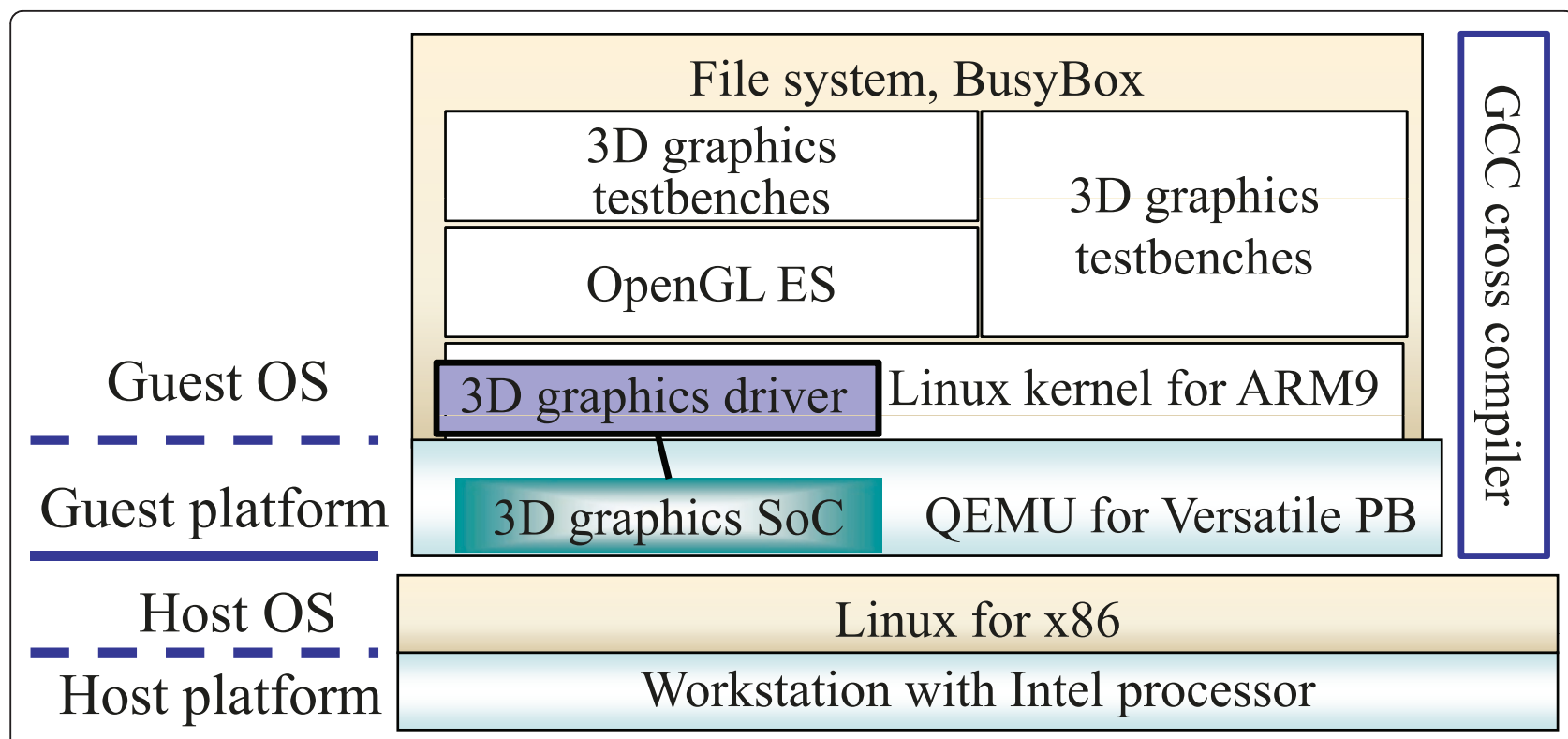

Figure 5 The software development environment for 3D graphics application.

Engine (RE) modules of the base addresses of these memory blocks.

By calling the mmap() function, the 3D graphics test benches can move 3D vertices into the 3D vertex buffer. After configuring the context table (CT) of the GE, the test benches will enable the GE to start 3D graphics operations. The CT contains the control registers of the $\mathrm{GE}$, while the control registers for the RE are collectively known as the register table (RT).

The 3D graphics SoC provides two interrupt signals that represent the GE and RE, respectively, shown at the bottom of Figure 4 . When the interrupt service routine (ISR) in the device driver receives the GE IRQ, this function will pull down the GE interrupt request (IRQ), and start the RE to continue the operation immediately.

The RE stores its results in the 32-bit temporary frame buffer, and then raises an IRQ to notify the ISR of $3 \mathrm{D}$ graphics device driver to truncate the pixel data from 32- to 16-bits. The truncation is necessitated by the difference in bit width between the development platform and the 3D graphics SoC. Finally, the 3D graphics object is displayed on the output screen.

\section{D graphics test benches}

The software designers development two types of 3D graphics test benches. The first type of test benches accesses 3D graphics device driver directly and owns only one object per frame. We have four 3D graphics test benches as shown in Table 3. The first two rows show the images in the test benches, and the last row gives the complexity of these test benches. Each vertex consists of 10 words. Each test bench has four modes, namely, the GE mode, RE mode, single frame mode, and multi-frame mode. The other type of test benches

Table 2 Corresponding configuration list of software development

\begin{tabular}{|c|c|}
\hline Components & Requirement \\
\hline Internet IO interface & $\begin{array}{l}\text { Address: 0xc230_0000, } \\
\text { Size: 0x80_0000, IRQ:29 and } 30\end{array}$ \\
\hline Linux kernel for ARM & 2.6.28-6 for Versatile PB \\
\hline Cross-platform compiler & GCC 3.4 \\
\hline root file system & $\begin{array}{l}\text { - Support ext2 file system } \\
\text { - Adopt BusyBox for Linux utilities }\end{array}$ \\
\hline Device driver & $\begin{array}{l}\text { - Initiate 3D graphics SoC } \\
\text { - Map physical memory address to logical memory address } \\
\text { - Handle interrupt service routine } \\
\text { - Truncate buffer data }\end{array}$ \\
\hline API & OpenGL ES 1.x or 2.x \\
\hline 3D graphics test program & Four test benches for 3D graphics SoC \\
\hline
\end{tabular}




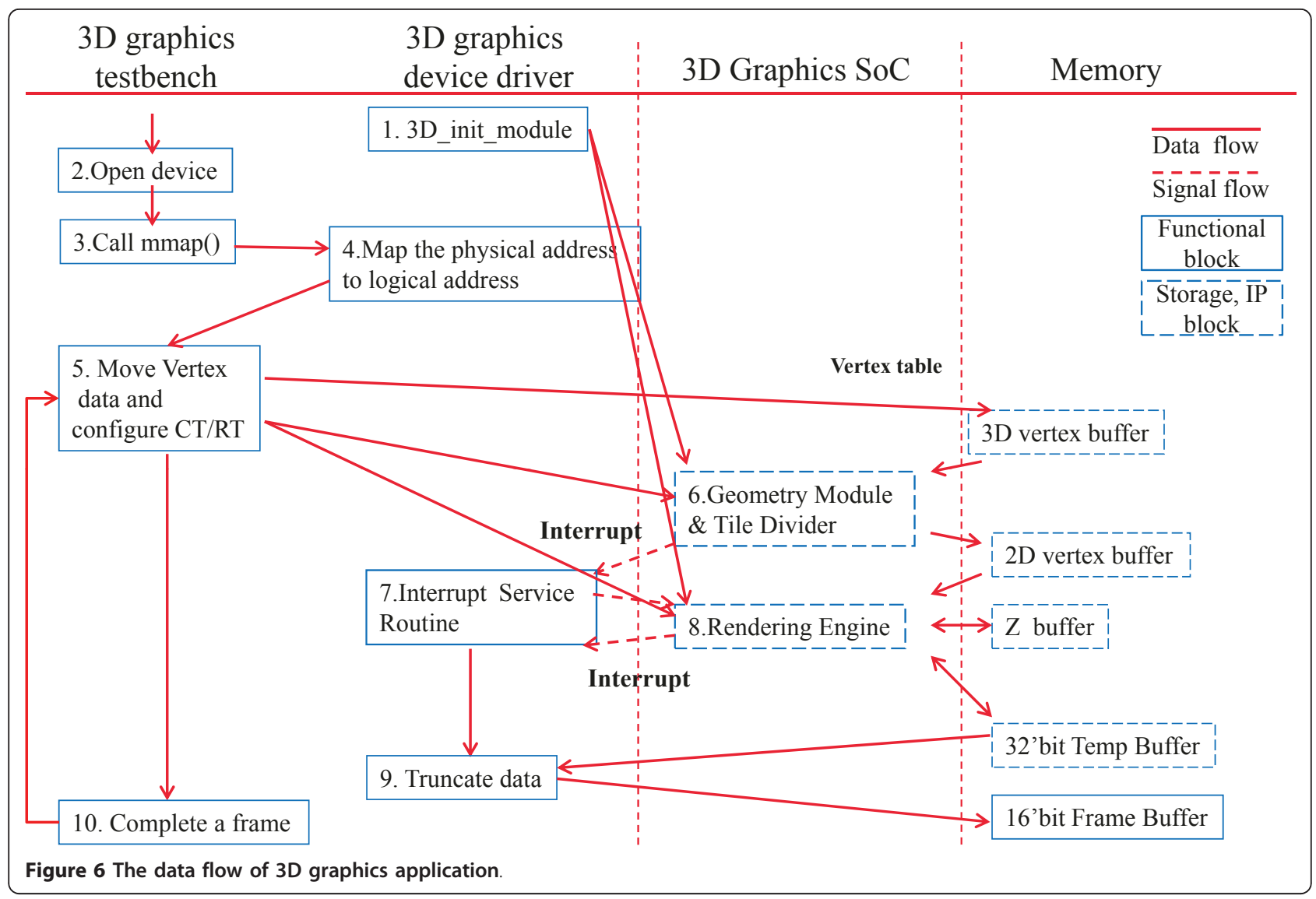

cooperates with OpenGL ES 1.x [28] and owns multiple objects per frame, as shown in Table 4.

\section{The QEMU interface}

This article leverages the QEMU VM to replace the Versatile $\mathrm{PB}$, thereby facilitating hardware and software co-design. The software stack used on top of the QEMU is shown in Figure 5. The IO interface in the QEMU is similar to the AMBA High-performance Bus (AHB) system bus that connects the Versatile PB and the FPGA. The latter implements the 3D graphics SoC hardware. This interface is part of the IO interface on the QEMU, as shown on the left-hand side of Figure 7.

Table 3 Four 3D graphics test benches

\begin{tabular}{lcccc}
\hline & & & & \\
\hline Testbenches & Triangle & Box & Cube & Teapot \\
\hline Vertex number & 3 & 36 & 144 & 18,960 \\
\hline
\end{tabular}

The QEMU provides two functions to support master $\mathrm{read} / \mathrm{write}$ operations. We also considered interrupt handling, and slave read/write operations from the slave interface, used by the 3D graphics SoC. After considering

Table 4 Experimental results of six 3D graphics testbenches with OpenGL ES 1.x

\begin{tabular}{|c|c|c|c|c|}
\hline & & Singapore & Taiwan & Ratio \\
\hline \multicolumn{2}{|c|}{ GM Operation Time } & 7,000 & 7,000 & 1.00 \\
\hline \multicolumn{2}{|c|}{ GM Data Idle } & $148,716,120$ & $16,925,520$ & 8.79 \\
\hline \multicolumn{2}{|c|}{ GM Wrapper Active } & $157,076,520$ & $18,584,060$ & 8.45 \\
\hline \multicolumn{2}{|c|}{ GM Wrapper Time } & $160,767,200$ & $18,700,440$ & 8.60 \\
\hline \multicolumn{2}{|c|}{ RE Operation Time } & $26,054,220$ & $2,253,390$ & 11.56 \\
\hline \multicolumn{2}{|c|}{ RE Data Idle } & $8,435,329,980$ & $649,543,730$ & 12.99 \\
\hline \multicolumn{2}{|c|}{ RE Wrapper Active } & $8,495,771,060$ & $653,808,750$ & 12.99 \\
\hline \multicolumn{2}{|c|}{ RE Wrapper Time } & $8,495,839,140$ & $653,876,830$ & 12.99 \\
\hline \multirow[t]{2}{*}{ GE (times) } & Receive & \multicolumn{2}{|c|}{516} & \\
\hline & Send & \multicolumn{2}{|c|}{604} & \\
\hline \multirow[t]{2}{*}{ RE (times) } & Receive & \multicolumn{2}{|c|}{26,003} & \\
\hline & Send & \multicolumn{2}{|c|}{51,385} & \\
\hline \multicolumn{2}{|c|}{ Total simulation time } & $1,808 \mathrm{~s}$ & $148 \mathrm{~s}$ & 12.22 \\
\hline
\end{tabular}




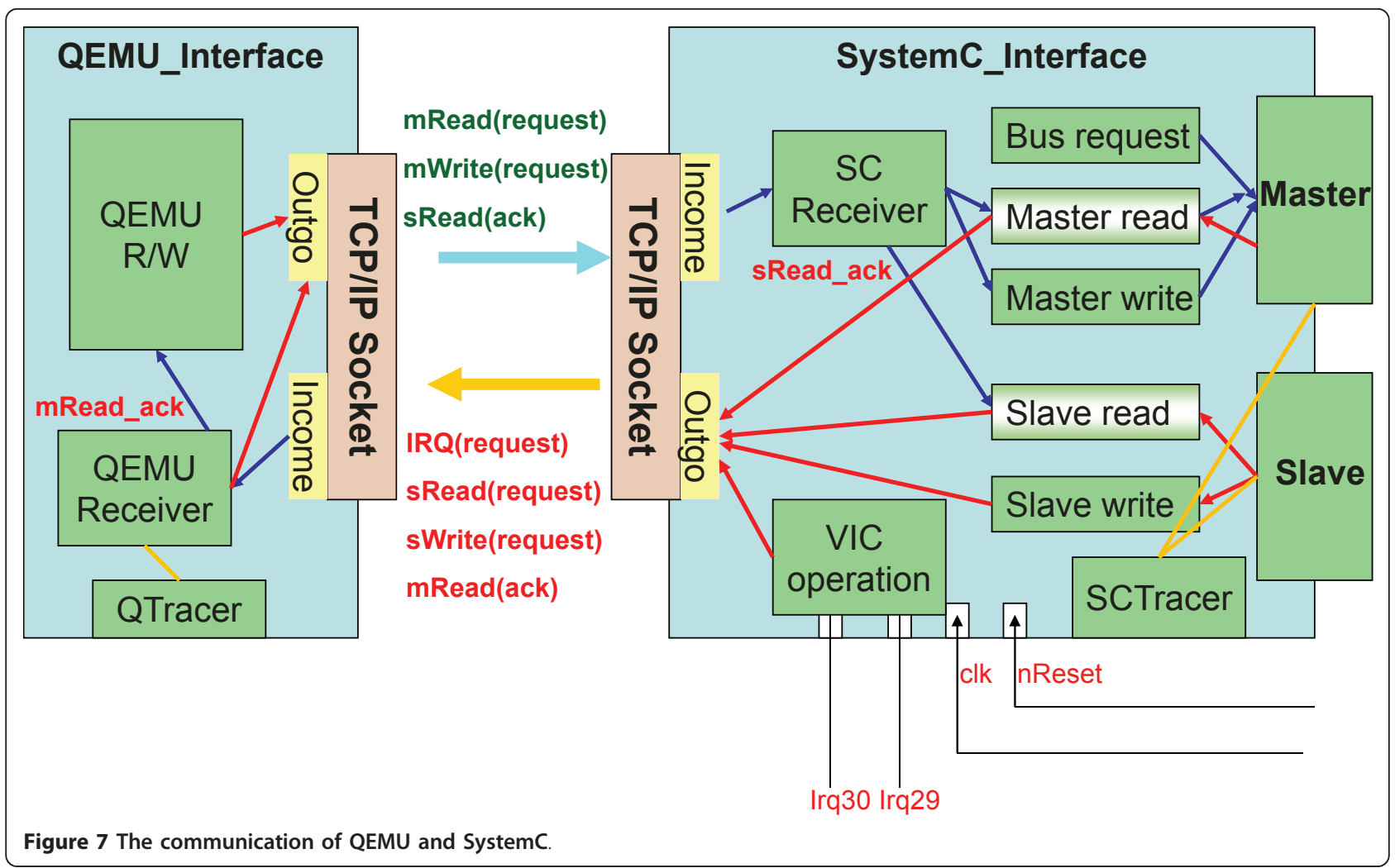

all the necessary operations, we defined two directions of communication consisting of seven types of operations, as shown in Figure 7.

The QEMU interface has three threads that allow it to handle different tasks. The QEMU R/W thread is a main thread provided by the QEMU in charge of processing the master read/write requests issued from the device driver. This thread will issue mReadRequest and mWriteRequest requests through an outgoing socket, and receives mReadAck acknowledgment through an incoming socket. The QEMU receiver thread receives any incoming network packages consisting of IRQRequest, sReadRequest, sWriteRequest, and mReadAck from the SystemC module shown on the right-hand side of Figure 7. The QEMU receiver acts as a slave interface and an interrupt controller. Depending on the content of the messages, it will read or write data from or to the QEMU, raise or pull down an interrupt signal, or pass mReadAck data to the QEMU R/W thread. The connection thread is responsible for maintaining the connection between the QEMU and the SystemC module.

For hardware/software co-verification, a QTracer module is embedded in the QEMU interface that stores data of the $2 \mathrm{D}$ coordinate vertices and frame buffer into a file. The framebuffer viewer is used to output the file, as shown in Figure 8. The diff command can also be used to compare the data to the expected log data [28].

\section{Communication interface and 3D graphics SOC}

This section introduces the hardware development environment of the proposed co-design framework consisting of two hardware parts. Figure 9 presents the block diagram of the full development environment. The first part is the QEMU and the authors create a virtual communication between the QEMU and the 3D graphics SoC. In principle, the software and hardware teams work concurrently, but in physical locations that are geographically dispersed. Therefore, the Internet is the best media for maintaining continuous communication between the teams. The second part is the 3D graphics SoC itself. This main part of the hardware design is implemented in SystemC using different abstraction levels.

\section{The SystemC interface}

The 3D graphics SoC is implemented in SystemC. The block diagram is shown on the left-hand side of Figure 9. We implemented a SystemC interface (SCI) to communicate with the QEMU via transmission control protocol/internet protocol (TCP/IP). The SCI acts as a TCP/ IP server that waits for the QEMU requests. The SCI 


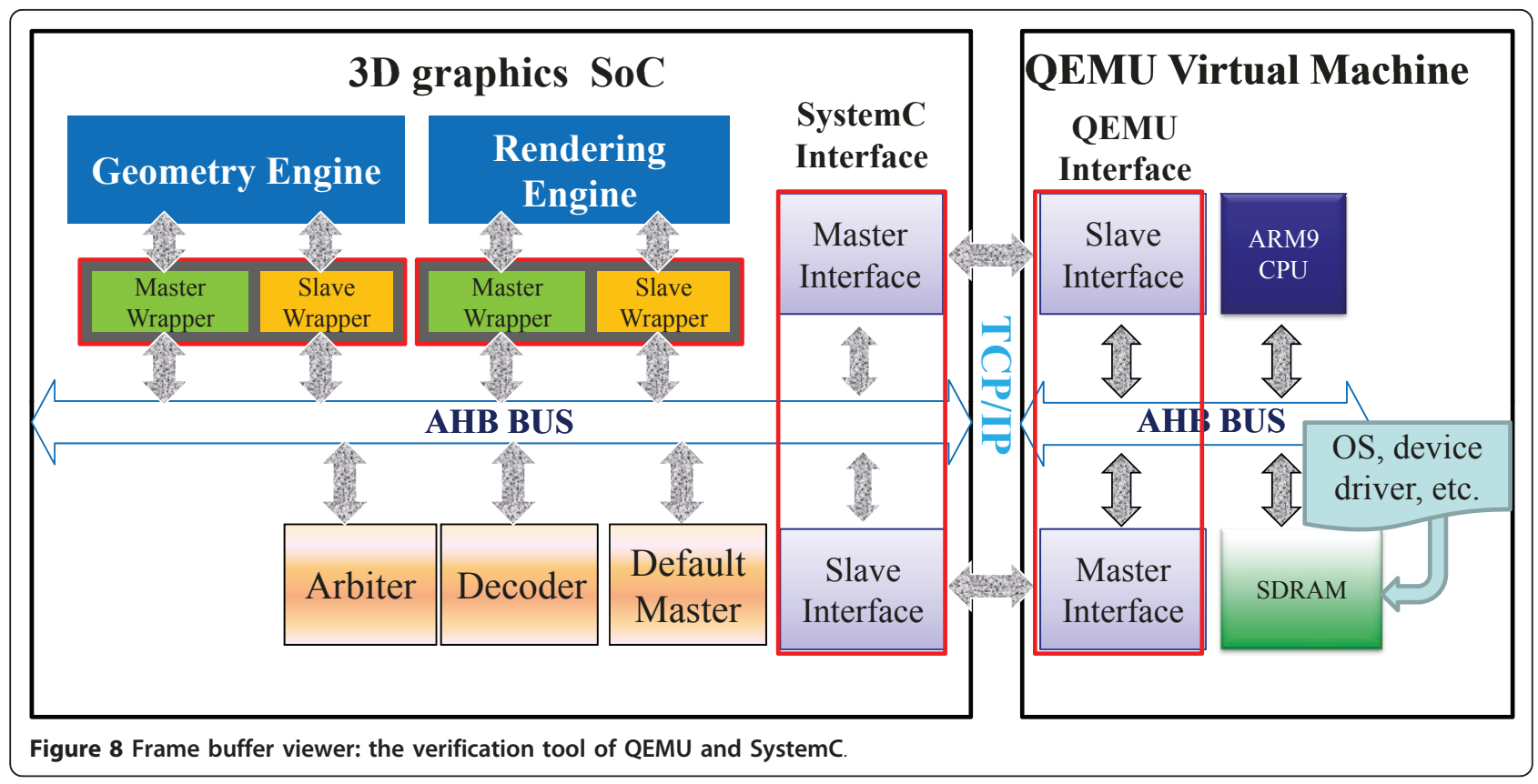

contains a master and a slave ports, as well as six main processes as shown on the right-hand side of Figure 7. The master read, master write, slave read, and slave write processes communicate with system bus. The SC receiver process gathers all incoming messages from the QEMU, and forwards them to the corresponding processes. The VIC operation process handles interrupts 29 and 30 from the $3 \mathrm{D}$ graphics SoC, while the SCTracer records the transactions on the master and slave ports. These can be reproduced later without full software execution. The SCTracer also stores the frame buffer in a file for checking. This will aid in offine debugging and architectural design space exploration.

Figure 10 shows the finite state machine (FSM) of the AHB master wrapper of the SCI. When the master wrapper receives a package, $\mathbf{Q}$, from the QEMU that contains an mRead or mWrite command, the FSM will transit from the Idle to the Request state. The SCI then calls the different functions provided by the AHB Library to process the master read or write command. To complete an mWrite operation, the SCI will notify the SC receiver that it has consumed the packet, and so the $\mathrm{SC}$ receiver may go ahead and receive another packet. For an mRead operation, the SCI enters the Read Ack state, and communicates the results of the read to the QEMU.

Figure 11 shows the FSM of the AHB slave wrapper in the SCI. The read data and write data states mean that AHB slave wrapper reads data from, or writes data to the QEMU. The SCI sends the packet to the QEMU for $\mathrm{read} / \mathrm{write}$ data via $\mathrm{TCP} / \mathrm{IP}$ on the Internet.
However, the state of read data needs to wait for the QEMU to send a read acknowledgement response.

\section{Two types of deadlock}

Figure 12 presents the static structure of the SCI, and then Figures 10 and 11 describe the dynamic state transition of the SCI according to the interaction of system operation. However, these figures are unable to detect certain problems, such as deadlocks. Some processes will hold resources, while at the same time requiring other resources held by others. The result is a deadlock. Figure 13 shows two types of deadlock that can happen in the SCI. The first type of deadlock happens when the QEMU wants to read data from the 3D graphics SoC, while at the same time, the $3 \mathrm{D}$ graphics SoC also wants to access the QEMU's memory. The master read process in the SCI holds both the locks for the QEMU readwrite in the QEMU interface, and the $S C$ receiver. At the same time, a slave read process requires the SC receiver to return data from the QEMU, and has successfully locked the AHB bus, resulting in a deadlock. According to our 3D graphics test benches, the master read process usually requires the values of the registers in the $3 \mathrm{D}$ graphics SoC.

However, this happens less frequently than the slave read process needs to read data from the $3 \mathrm{D}$ graphics SoC. Therefore, to resolve this deadlock, we force the master read process to give up the SC receiver. The SC receiver will record the master read request, and rerequest the AHB bus when it is idle. The QEMU will be suspended until this happens. 


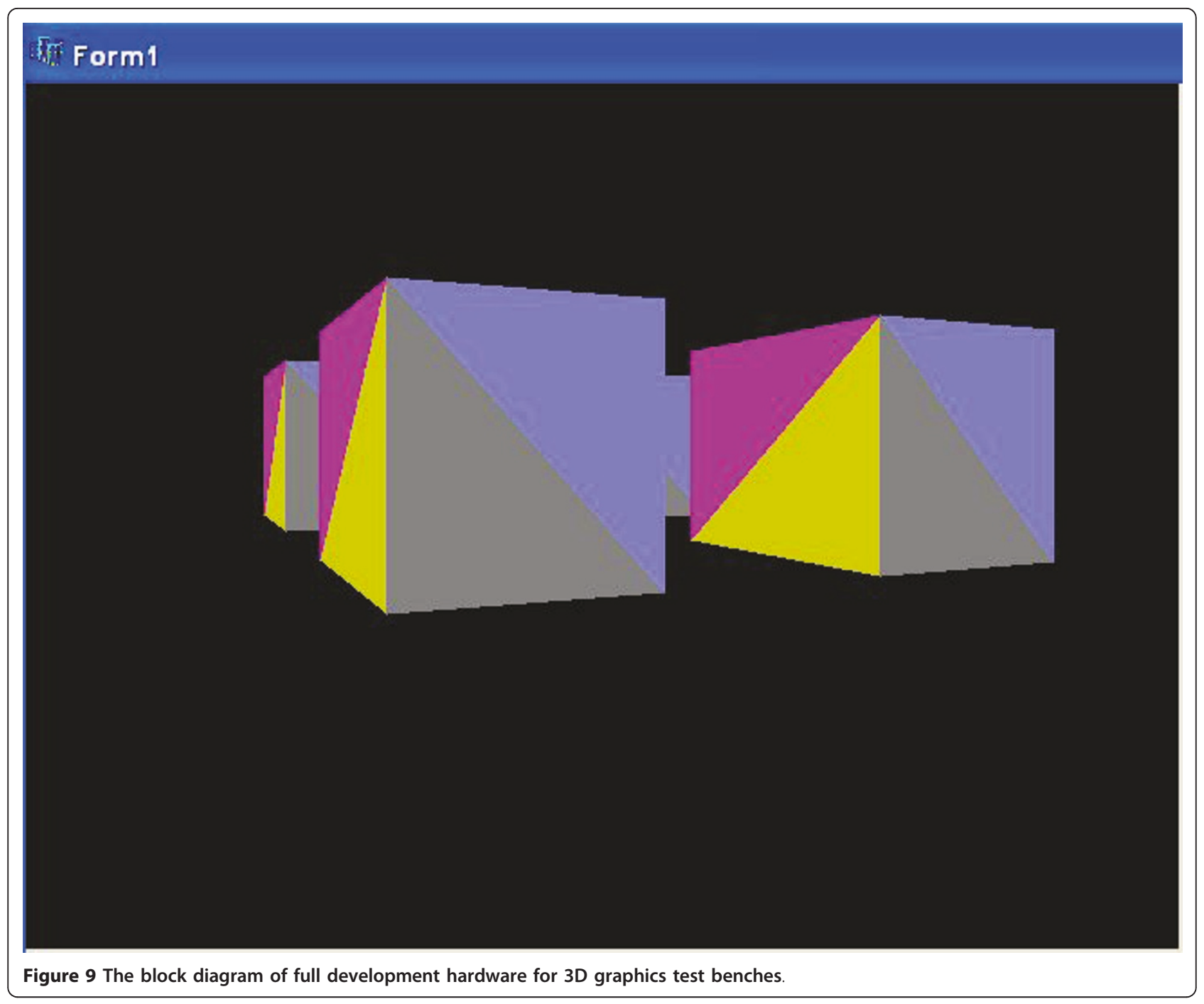

The second type of deadlock is shown at the bottom of Figure 13. During the time when the application is writing to the control registers, the $3 \mathrm{D}$ graphics SoC may concurrently attempt to read data from the memory of the QEMU. A deadlock occurs as the master write process is unable to lock the bus, but the slave read is unable to complete its data request via the $\mathrm{SC}$ receiver. We store the request of the master write process into a cyclic queue to solve this deadlock, because the master write process does not wait for an acknowledgement. This solution results in a race condition between the $\mathrm{SC}$ receiver and the master write process as the $\mathrm{SC}$ receiver attempts to enqueue while the master write process attempts to dequeue. We solve this problem by making the operations atomic.

\section{The 3D graphics SoC}

To take the trade-off between simulation time and accuracy into account, the $3 \mathrm{D}$ graphics SoC mixes two types of
TLM accuracy, namely time-approximate accuracy and bus cycle accuracy (BCA). Time-approximate accuracy of TLM evaluates time information accurately but does not simulate the cycle count actually. BCA is triggered by a cycle event and takes more time than the time-approximate model. The computation of the GE and RE [29] uses time-approximate accuracy. This saves simulation time and provides accurate enough cycle information to hardware designers for architecture analysis. Each IP connected to the AHB uses BCA, simulated by Platform Architect from Synopsys, Inc [15]. For instance, the authors gather the time information of the geometry module (GM) operation to understand the effects of the complexity of the 3D graphics operation. However, they analyze the latency of GM master wrapper to determine the impact of the bus architecture, shown in Figure 14.

The GE comprises two main functional modules: a GM and a tile divider module (TDM). The input data of 

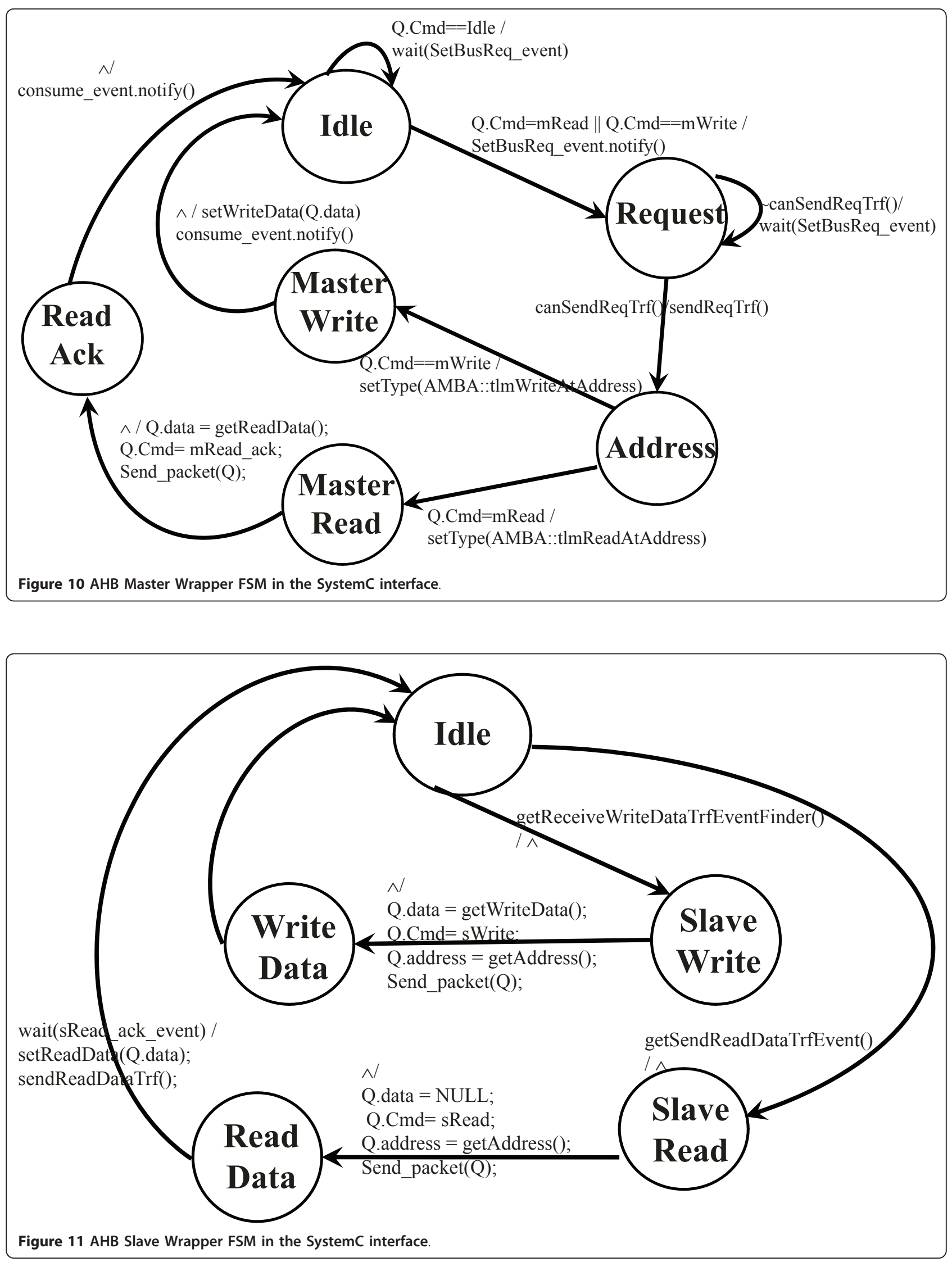

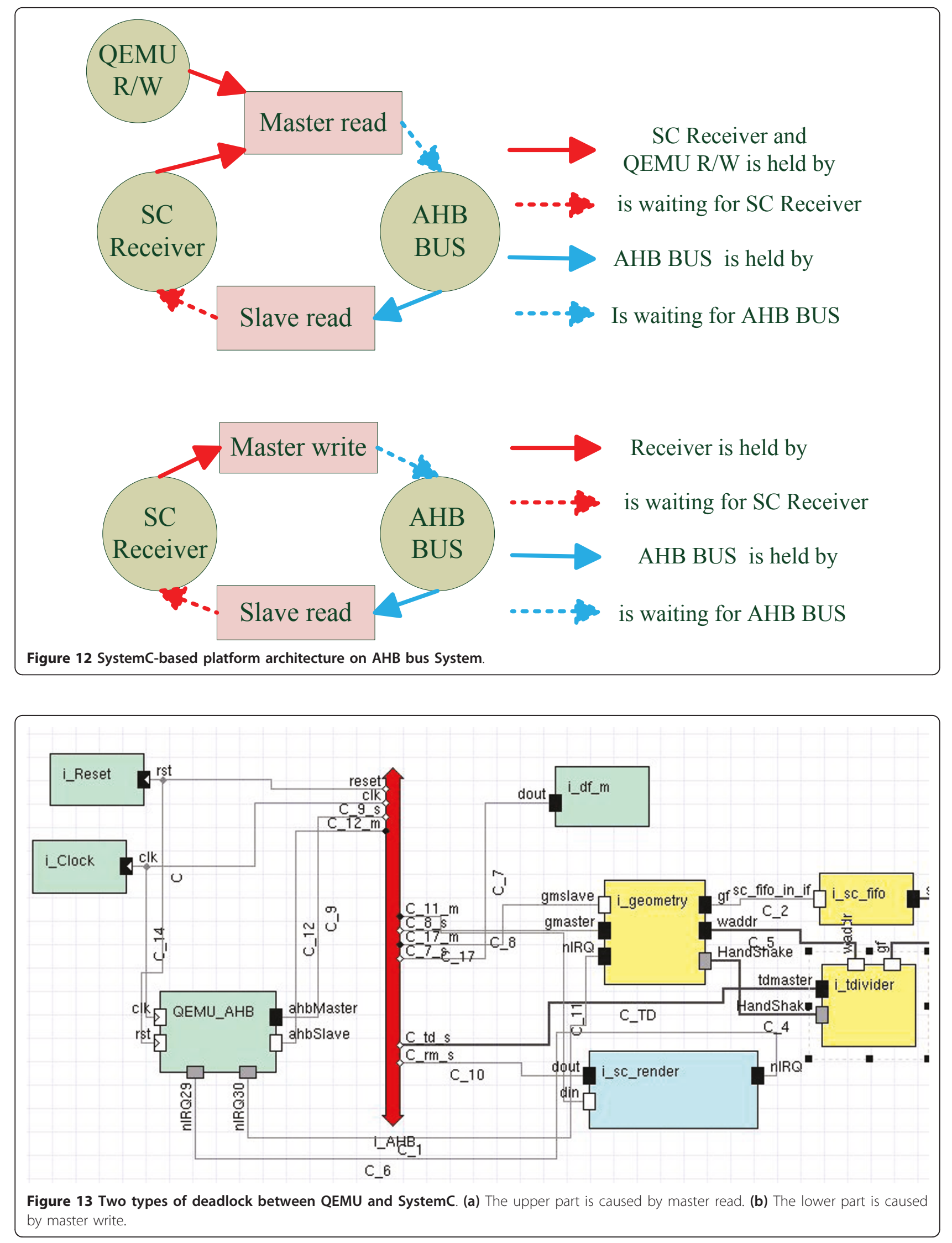


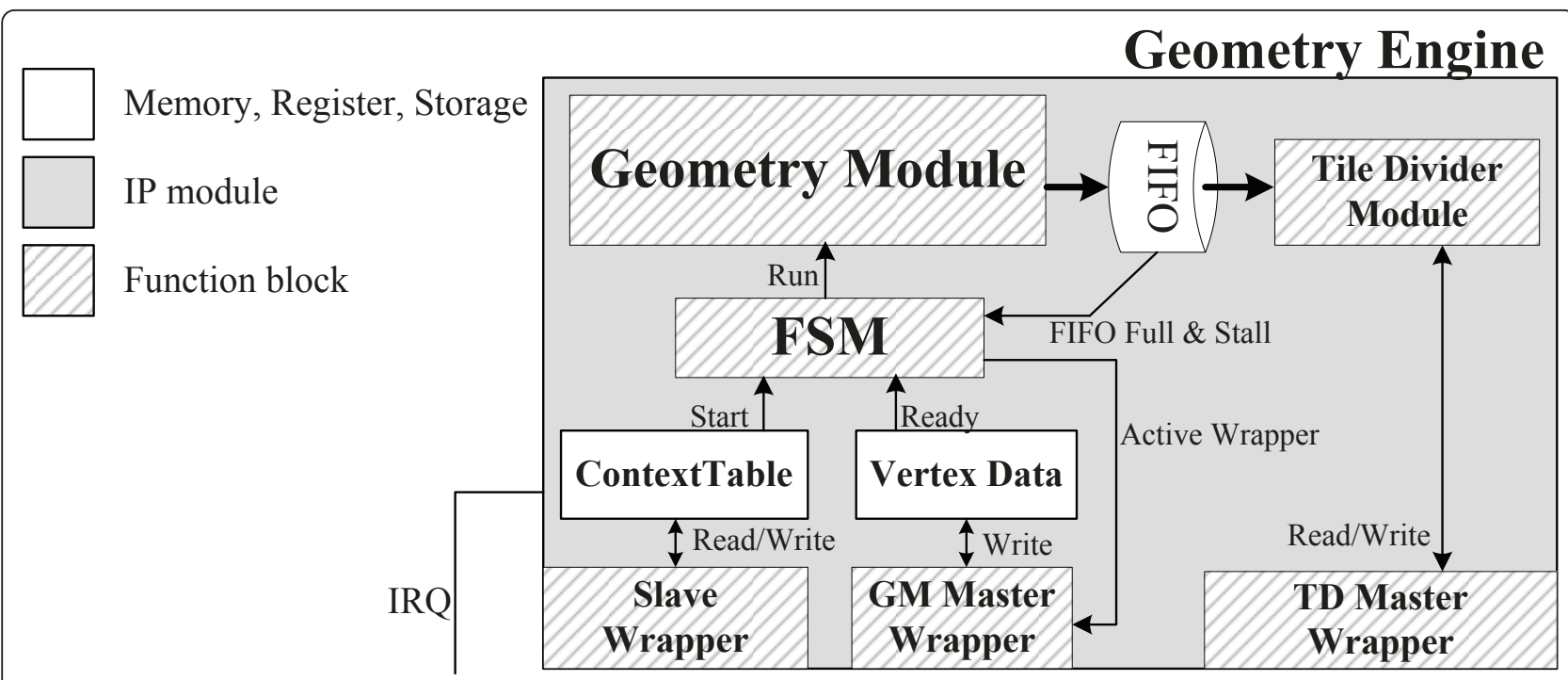

Figure 14 Block diagram of geometry engine [29].

the GE is from the 3D vertex buffer and output data is sent to the $2 \mathrm{D}$ vertex buffer in main memory. The GE provides a slave wrapper, a GM master wrapper, a tile divider (TD) master wrapper, and an interrupt signal in its interface. The processor configures geometry operation registers (the $\mathrm{CT}$ ) via the slave wrapper of the GE. The GM master wrapper provides data for the internal GM read from the 3D vertex buffer. The TD master wrapper is dedicated to handle the $2 \mathrm{D}$ vertex buffer. When the GE finishes its assigned task, it notifies the QEMU through an interrupt signal.

The GM is responsible for the transformation and lighting, culling, and clipping of 3D graphics geometry operations. The TDM is implemented using a tile-based concept [30] so as to reduce the number of memory accesses. The TDM creates tile list data in the $2 \mathrm{D}$ vertex buffer for the RE. Output data is passed to the tile divider module, which builds a tiled triangle list for the RE. The GM has three pipeline stages, each about 16 cycles in length. A first-in-first-out (FIFO) buffer is needed between the GM and the TDM because of their different speeds. If the FIFO buffer becomes full, then the GM will stall.

The RE consists of three functional modules: the triangle setup, the rasterizer, and the per-fragment operations modules, shown in Figure 15. It also adopts a tilebased approach so as to reduce the memory bandwidth. We used a tile size of $32 \times 32$ pixels, which generally yields the best trade-off between the amount of on-chip memory and the amount of external data traffic. The RE handles three types of data: the $2 \mathrm{D}$ vertex buffer, the frame buffer, and the Z/Stencil buffer. The QEMU configures the RE's RT through its slave wrapper. The RE reads data from the $2 \mathrm{D}$ vertex buffer and outputs the results to the frame buffer and the Z/Stencil buffer. The frame buffer stores pixel values, and the Z/Stencil buffer stores the $\mathrm{Z} /$ Stencil values. The implementation is designed by the Synopsys EDA tool, shown in Figure 12.

\section{Experiment results}

The authors validated their proposed framework in two methods, both for feasibility and reproducibility. The first method is to develop the different applications using the proposed environment, and the other is to reproduce the software development environment at several places. At first, they applied the proposed environment to the different applications, a 3D graphics application and a JPEG decoder, using the general communication. There did not have any modification on the SCI and the QEMU interface for these applications.

Then, the authors examine two experiments using the application-specific communication. At first, they successfully reproduced the software development environment at seven locations and five countries around the world. The second experiment is to validate the feasibility of 3D graphics application and the authors execute six test benches with OpenGL ES API.

\section{General communication}

Table 5 shows the simulation result of the proposed framework when developing software in Singapore and Taiwan laboratories separately, while the hardware design is held in a Taiwanese laboratory. The first row represents the locations of developing software. The QEMU will transfers a single command package in each transmission. Each package contains only three words, namely the command type, address, and data. The second to fifth rows of the table report the simulation results of the GM, and the next four rows are the results 


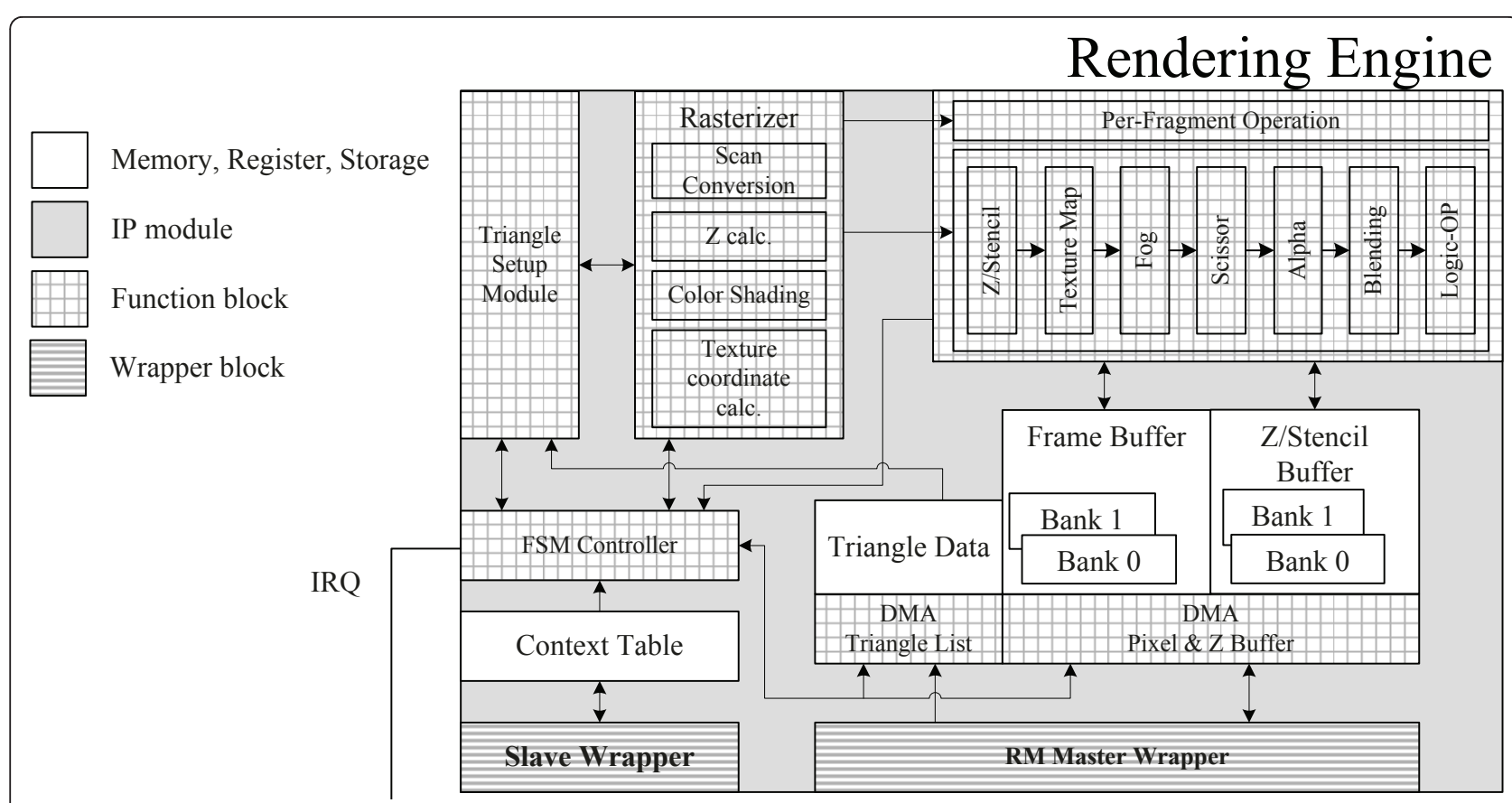

Figure 15 Block diagram of rendering engine [29].

of the RE. It is obvious that the GM is idle most of the time. The hardware/software co-simulation result is highly dependent on the speed of the network, and it is difficult to perform design space exploration over the network. Performing the experiments locally is $8 \times$ to $13 \times$ faster than doing it over the Internet. The ninth and tenth rows show the number of receive and send packages in the SCI. The screen size is $640 \times 480$, and the RE needs to access the frame and $\mathrm{Z}$ buffer. Therefore, the number of RE operations far exceed that of GE operations.

The general communication between the QEMU and $3 \mathrm{D}$ graphics SoC is transparent to the software/hardware designers and similar to that between the FPGA and the Versatile PB. Our lab tried to verify the functionality of the new physical development baseboard, Socle Leopard 6 SoC Design Platform [20]. The hardware designer implemented inverse discrete cosine transform (IDCT) [31], a part of JPEG decoder [32], at RTL and download it into its FPGA. Then the software designer wrote an IDCT device driver to transfer data to/from the FPGA. The software designer adopted the proposed development environment to build the SystemC and RTL cosimulation platform shown in Figure 16 as DUT part of Figure 4. The verification method is to compare the results extracted from IDCT at RTL to the golden

Table 5 The general communication between the QEMU and 3D graphics SoC using SystemC for test bench Box (measured unit: Cycle)

\begin{tabular}{cccc}
\hline & General Communication & Application-specific Communication & Speedup \\
\hline GM Operation Time & 7,000 & 7,120 & 0.98 \\
\hline GM Data Idle & $16,925,520$ & 2,050 & 8256.35 \\
\hline GM Wrapper Active & $18,584,060$ & 4,440 & 4185.60 \\
\hline GM Wrapper Time & $18,700,440$ & 6,110 & 3060.63 \\
\hline RE Operation Time & & & 132,330 \\
\hline RE Data Idle & $2,253,390$ & $2,129,280$ & 305.05 \\
\hline RE Wrapper Active & $649,543,730$ & $1,815,960$ & 360.03 \\
\hline RE Wrapper Time & $653,808,750$ & $1,984,060$ & 329.57 \\
\hline Total simulation time & $653,876,830$ & & 29.60 \\
\hline
\end{tabular}




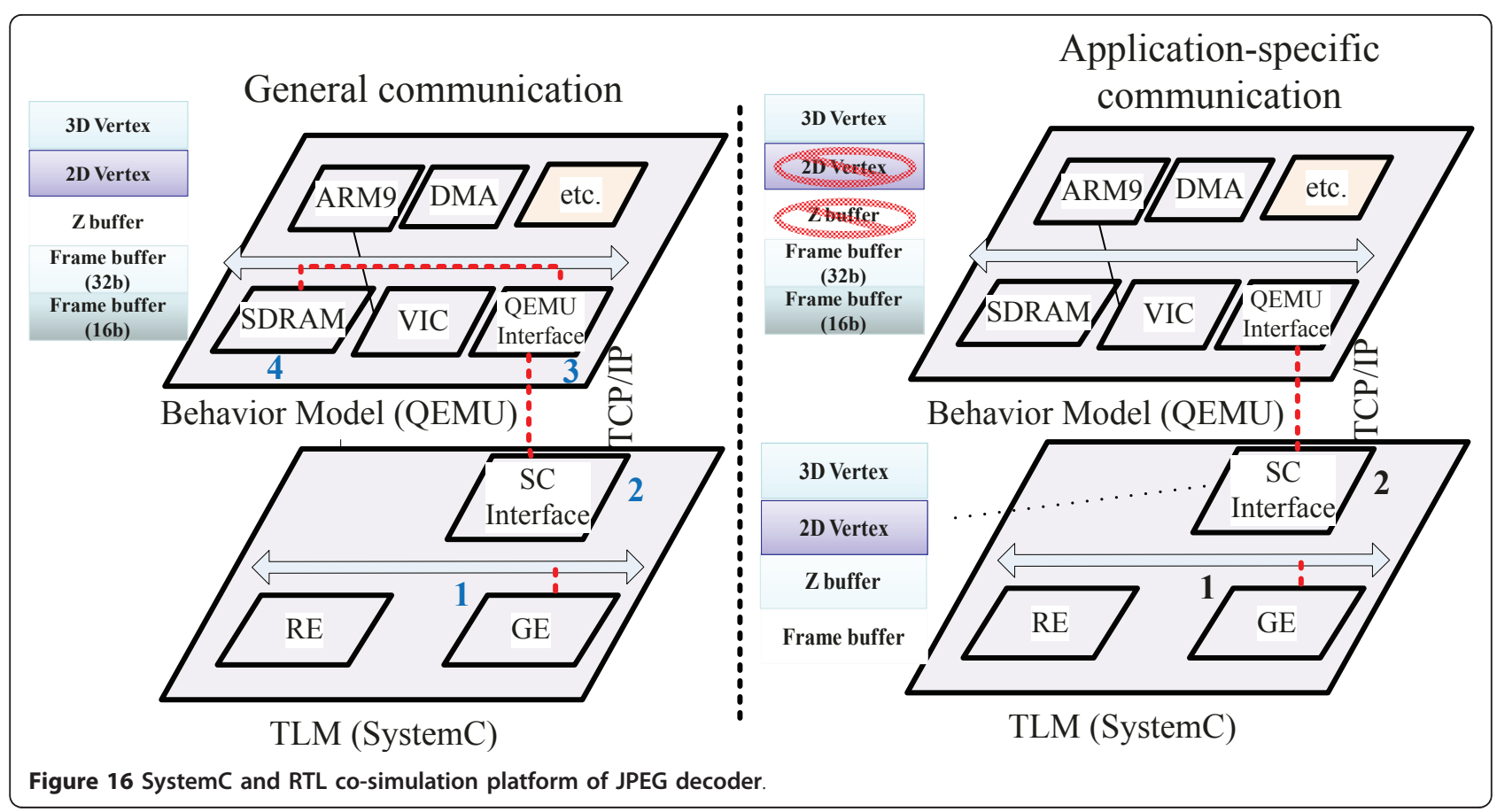

results generated from the IDCT software module. These designers did not have any knowledge and modification of the QEMU interface and the SCI.

However, the method cannot provide useful cycle information to the hardware designers. The variable evaluation results among the wrappers and execution time is useless for design space exploration. The other problem is the longer simulation time. To resolve this problem, this article proposes another communication, the application-specific communication, in the next section.

\section{Application-specific communication}

Figure 6 shows the data flow of the 3D graphics test benches. These test benches generate the $3 \mathrm{D}$ vertex data and configure to the GE/RE using the device driver at step 5. After that, GE and RE have numerous $R / W$ operations to different blocks, such as $3 \mathrm{D}$ vertex buffer, $2 \mathrm{D}$ vertex buffer, $\mathrm{Z}$ buffer, and 32'bit frame buffer, in main memory. These memory blocks are reserved for 3D graphics SoC, so no other else will access these blocks. The authors modify the SCI and QEMU interface to keep these operation within SystemC side. This modification will not affect the hardware and software design. This communication depends on the data flow of the target application, so it is called as the application-specific communication. Figure 17 shows the difference in the data flow between the general and the application-specific communication. For instance, when the GE writes data into synchronous dynamic random access memory (SDRAM) with general communication, the flow is as follows:

1. The GE writes data on the AHB bus;

2. The SCI receives data, and then forwards them via TCP/IP;

3. The QEMU interface writes data into the specified address;

4. The SDRAM receives the data.

The test bench Box owns 36 vertexes and each vertex occupies 10 words. It means the SCI interface sends 360 read requests to the QEMU at least while the RE at the bottom of Figure 17 reads 3D vertex data from 3D vertex buffer at the top of Figure 17 using the general communication. However, if the interface designers pack 3D vertex as a package and store them into the SCI as 3D vertex buffer, the only one transmission is needed. In the case of the application-specific communication, the SCI receives data from the GE and stores them into internal buffers. The hardware and software designers can use the application-specific communication without modifying their implementation.

The GE and RE consider the SCI not only as a test bench generator, but also as a memory module comprising of the $2 \mathrm{D}$ vertex buffer, the Z-buffer, and the frame buffer. When the RE triggers an interrupt signal to the SCI after completing a frame, the SCI moves all the data in the frame buffer to the QEMU interface. The QEMU interface notifies the device driver using interrupt 


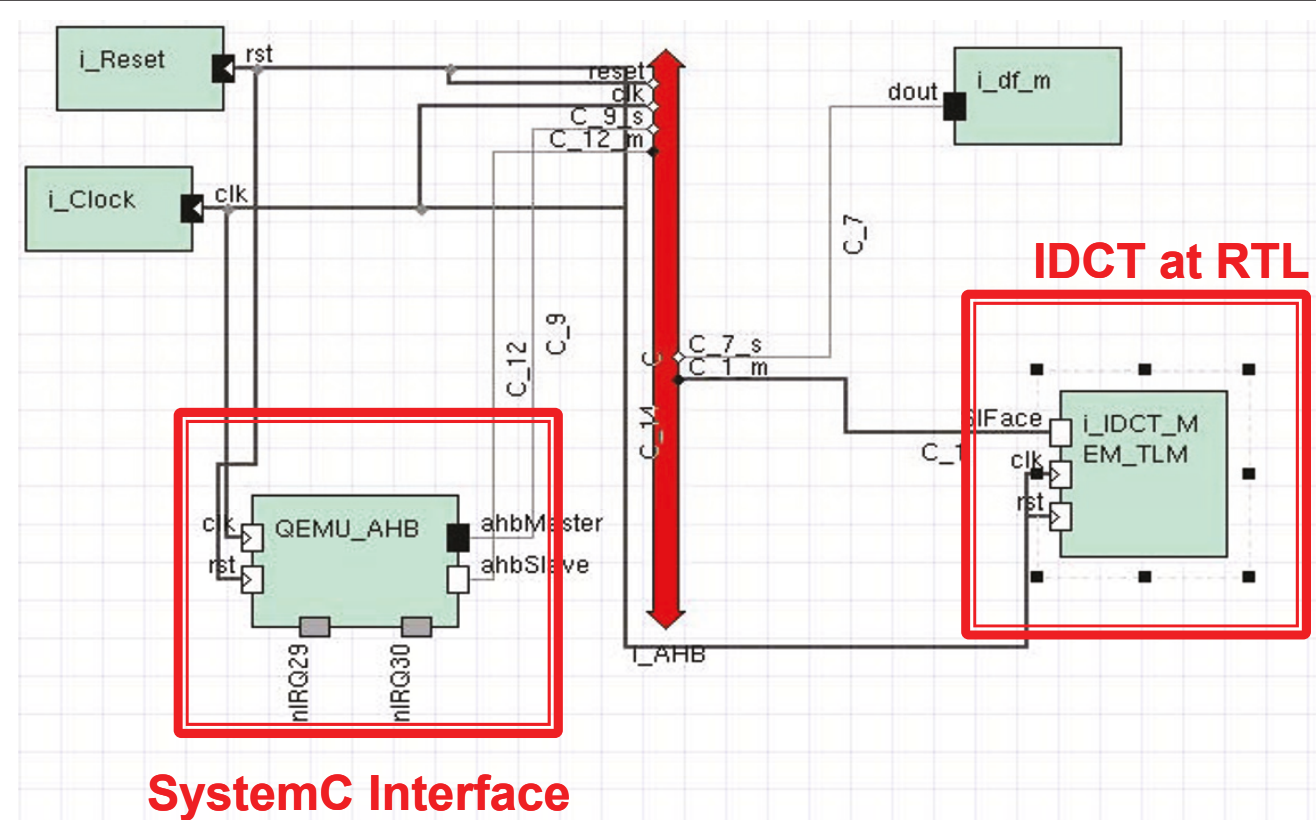

Figure 17 The difference of the data flow among general communication and application-specific communication.

function call on the QEMU platform. The 3D graphics device driver truncates the data from 32- to 16-bits to display them on the QEMU. This processing contains two massive data transmissions: the $3 \mathrm{D}$ vertex buffer from the QEMU interface to the SCI, and the frame buffer from the SCI to the QEMU interface. Performance is significantly improved. Table 6 shows the comparison between the general and the application-specific communication. The GM data idle time is reduced by over $8,256 \times$, and the total simulation time increased by $29 \times$. Notably, the hardware simulation cycle time is the same no matter where the software test benches are executed in. Because the GM waits for the TD to complete its operation, the time for the GM operation using the general communication is less than that using the application-specific communication.
To validate the reproducibility and feasibility of our framework, the authors encapsulated the entire software development environment as a VirtualBox [33] image file, and asked several volunteers at different countries $\mathrm{s}$ around the world, such as Singapore, Romania, Australia, England, and USA, to run these experiments. The development environment consists of software development and hardware development platform shown in the top of Figure 4a and also owns the four 3D graphics test benches listed in Table 3, with each test bench showing three different frames. The volunteers' environments act as TCP clients, and DUT in Taiwan acts as a TCP server. We provide not only the VirtualBox image also instruction document on the Internet to the volunteers to execute the instructions step by step. Table 7 shows the results of the geographic differences measured by

Table 6 Comparison of general and application-specific communication (in cycles) for Box testbench on an entirely local setup

\begin{tabular}{lcccc}
\hline Location of Software Design & Triangle & Box & Cube & Teapot \\
\hline LAN & $9 / 5 / 5$ & $9 / 5 / 5$ & $14 / 11 / 11$ & $30 / 27 / 26$ \\
\hline Taiwan & $10 / 5 / 5$ & $11 / 6 / 5$ & $15 / 15 / 15$ & $55 / 52 / 48$ \\
\hline Singapore & $20 / 11 / 15$ & $20 / 15 / 15$ & $20 / 20 / 20$ & $41 / 40 / 36$ \\
\hline Romania & $150 / 151 / 151$ & $140 / 141 / 145$ & $136 / 136 / 132$ & $174 / 164 / 165$ \\
\hline Australia & $20 / 15 / 15$ & $16 / 10 / 10$ & $25 / 25 / 25$ & $50 / 44 / 43$ \\
\hline London & $16 / 10 / 10$ & $15 / 11 / 10$ & $21 / 20 / 20$ & $46 / 46 / 44$ \\
\hline North Carolina, USA & $30 / 30 / 25$ & $30 / 30 / 30$ & $36 / 35 / 31$ & $62 / 54 / 60$ \\
\hline Eugene, OR, USA & $15 / 10 / 10$ & $15 / 10 / 10$ & $20 / 15 / 15$ & $41 / 34 / 35$ \\
\hline San Diego, CA, USA & $15 / 15 / 10$ & $15 / 10 / 10$ & $15 / 15 / 20$ & $43 / 35 / 35$ \\
\hline
\end{tabular}


second. The third row, 'Taiwan,' means that the software design and the hardware design are on different network segments in Taiwan, while 'LAN' refers to an entirely local area network setup. The first three test benches own small number of vertexes, so they send the similar number of packages. The latency and stability of the Internet cause the deviation of simulation time.

Our lab have already developed the OpenGL ES 1.x working with our 3D graphics SoC on Versatile PB [28]. The authors migrated these 3D graphics test benches from Versatile PB to our proposed Internet-based platform and adjusted the QEMU interface and the SCI to comply with the data flow of these test benches. The results of the six 3D graphics test benches are shown in Table 4. The "Object" field shows the number of objects for each frame, and the "Vertex" field gives the number of the first object that represents the complexity of these testbenches. Finally, the "Time" field presents the simulation time in second using the application-specific communication.

\section{Conclusion}

This article proposed an Internet-based hardware/software co-design framework. The authors have successfully applied this framework to develop a 3D graphics system-on-a-chip hardware, and the full software stack necessary to use this hardware. Our integrated framework achieved the following goals: (1) it provides a full system simulation that includes the hardware as well as the software system; (2) it permits a very early start of concurrent hardware and software development; (3) it

Table 7 Experimental results (in seconds) when reproducing at the different places

\begin{tabular}{|c|c|c|c|}
\hline \multicolumn{2}{|c|}{ Cube } & \multicolumn{2}{|c|}{ Door } \\
\hline Object & 3 & Object & 1 \\
\hline Vertex & 6 & Vertex & 1644 \\
\hline Time & 14.5 & Time & 2.5 \\
\hline \multicolumn{4}{|c|}{ 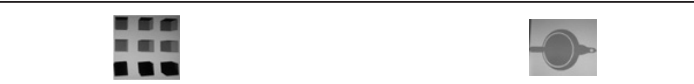 } \\
\hline \multicolumn{2}{|c|}{ Multiple cube } & \multicolumn{2}{|c|}{ Teapot } \\
\hline Object & 54 & Object & 7 \\
\hline Vertex & 6 & Vertex & 3000 \\
\hline Time & 267.2 & Time & 37.7 \\
\hline \multicolumn{2}{|c|}{ Beethoven } & \multicolumn{2}{|c|}{ Castle } \\
\hline Object & 31 & Object & 36 \\
\hline Vertex & 3000 & Vertex & 3000 \\
\hline Time & 153.1 & Time & 182.5 \\
\hline
\end{tabular}

reproduces various test benches of 3D graphics development by means of the SCTracer; (4) it reproduces the software environment at different places around the world; (5) it adopts application-specific communication to avoid the effect of network transactions achieving a $29.6 \times$ increase in speed when compared to general communication; and (6) it seamlessly migrates the different abstraction levels, TLM/RTL/FPGA. An important property of this framework is that someone in a different part of the development process can execute and reproduce the functionality of another part of the design that was implemented in a geographically remote location. Furthermore, such sharing can be done in a controlled and secure manner. The authors believe that this will significantly increase the productivity of the design teams as a whole.

\section{Abbreviations}

AHB: AMBA High-performance Bus; API: application programming interface; BCA: bus cycle accuracy; CT: context table; DUT: design-under-test; DUT: design-under-test; EDA: electronic design automation; ESL: electronic systemlevel; FPGA: field programmable gate array; FSM: finite state machine; FIFO: first-in-first-out; GE: geometry engine; GM: geometry module; GPSs: global positioning systems; GCC: GNU Compiler Collection; IP: intellectual property; IRQ: interrupt request; ISR: interrupt service routine; IDCT: inverse discrete cosine transform; OS: operating system; PDAs: personal digital assistants; PB: platform baseboard; RT: register table; RTL: register transfer level; RE: rendering engine; SSL: secure sockets layer; SDRAM: synchronous dynamic random access memory; SCl: SystemC interface; SLDLs: system-level design languages; SoC: system-on-a-chip; 3D, three-dimensional; TD: tile divider; TDM: tile divider module; TLM: transaction-level modeling; TCP/IP:

transmission control protocol/internet protocol; VM: virtual machine; VPN: virtual private network

\section{Acknowledgements}

The authors would like to thank Himax Technologies Inc. for partially sponsoring our research and thank Professor Chung-Ho Chen of NCKU and his research team for helping the set up of the initial QEMU-SystemC environment. We are also indebted to the volunteers from around the world, including HueySiang Kam in Australia, Mariam Sood in England, Ioana Hagiescu in Romania, Bipasa, CheeWai Lee, and Ding Yang in USA, Wen Chiou in Taiwan, for testing and reproducing our work. This work was partially supported by National Science Council (NSC) of Taiwan, R.O.C. under contrasts NSC 99-2220-E-110-008 and NSC98-2917-I-110-101.

\section{Author details}

${ }^{1}$ Department of Computer Science and Engineering, National Sun Yat-Sen University, Kaohsiung, Taiwan ${ }^{2}$ Department of Information Management, Shih Chien University, Taiwan ${ }^{3}$ Department of Computer Science, National University of Singapore, Singapore 117417

\section{Competing interests}

The authors declare that they have no competing interests.

Received: 1 November 2010 Accepted: 19 July 2011

Published: 19 July 2011

\section{References}

1. B Bailey, GE Martin, A Piziali, ESL design and verification: a prescription for electronic system-level methodology (Morgan Kaufmann, 2007)

2. Open SystemC Initiative (OSCI). http://www.systemc.org/home/

3. L Cai, D Gajski, Transaction Level Modeling: An Overview. in Proc of First IEEE/ACM/IFIP International Conference on Hardware/Software Codesign and System Synthesis, 19-24 (2003) 
4. S Pasricha, N Dutt, M Ben-Romdhane, Extending the Transaction Level Modeling Approach for Fast Communication Architecture Exploration. in Proc of 41st Design Automation Conference, 113-118 (2004)

5. T Grötker, S Liao, G Martin, S Swan, System Design with SystemC. (Kluwer Academic, Boston, 2002)

6. D Gajski, J Zhu, R Dömer, A Gerstlauer, S Zhao, SpecC: Specification Language and Methodology. (Kluwer Academic, 2000)

7. R Walstrom, J Schneider, D Rover, Teaching system-level design using SpecC and SystemC. in Microelectronic Systems Education, 2005. (MSE '05). Proceedings. 2005 IEEE International Conference on, 95-96 (2005)

8. T Kogel, A Wieferink, H Meyr, A Kroll, SystemC Based Architecture Exploration of a 3D Graphic Processor. in Proc IEEE Workshop on Signal Processing Systems, 169-176 (2001)

9. D Crisu, S Cotofana, S Vassiliadis, A Hardware/Software Co-Simulation Environment for Graphics Accelerator Development in ARM-Based SoCs. in Proc 13th Annual Workshop on Circuits, Systems and Signal Processing (ProRISC'02), 255-268 (2002)

10. B Juurlink, I Antochi, D Crisu, S Cotofana, S Vassiliadis, GRAAL: A Framework for Low-Power 3D Graphics Accelerators. IEEE Comput Graph Appl. 28(4), 63-73 (2008)

11. M Rupp, A Burg, E Beck, Rapid prototyping for wireless designs: the fiveones approach. Signal Processing. 83(7), 1427-1444 (2003). doi:10.1016/ S0165-1684(03)00090-2

12. R Rajagopal, S Ramamoorthy, L Wenzel, H Andrade, A Rapid Prototyping Tool for Embedded, Real-Time Hierarchical Control Systems. EURASIP Journal on Embedded Systems 2008. 14 (2008)

13. J Buck, S Ha, EA Lee, DG Messerschmitt, Ptolemy: A Framework for Simulating and Prototyping Heterogenous Systems. International Journal in Computer Simulation. 4(2), 1-34 (1994)

14. M Pelcat, J Piat, M Wipliez, S Aridhi, J-F Nezan, An Open Framework for Rapid Prototyping of Signal Processing Applications. EURASIP Journal on Embedded Systems 2009. 13 (2009)

15. Synopsys platform architect. http://www.synopsys.com/Systems/ ArchitectureDesign/Pages/PlatformArchitect.aspx

16. Synopsys galaxy implementation platform. http://www.synopsys.com/ Solutions/EndSolutions/Galaxylmplementation/Pages/default.aspx

17. Synopsys milkyway database. http://www.synopsys.com/solutions/ endsolutions/galaxyimplementation/pages/milkyway.aspx

18. P Belanovic, An open tool integration environment for efficient design of embedded systems in wireless communications, PhD thesis, Technische Universität Wien, Wien, Austria. (2006)

19. Versatile Platform Baseboard for ARM926EJ-STM[online]. http://www.arm. com/products/tools/development-boards/versatile/index.php

20. Socle Leopard 6 SoC Design Platform. http://www.socle-tech.com.tw/en/ service $62 . \mathrm{html}$

21. QEMU. http://wiki.qemu.org/Index.html

22. JW Lin, CC Wang, CY Chang, CH Chen, KJ Lee, YH Chu, JC Yeh, YC Hsiao, Full System Simulation and Verification Framework, in Information Assurance and Security, 2009. IAS '09 Fifth International Conference on. 1, 165-168 (2009)

23. QEMU-SystemC, greensocs. http://www.greensocs.com/en/projects/ QEMUSystemC

24. GCC, the GNU Compiler Collection. http://gcc.gnu.org/

25. The Linux Kernel Archives. http://www.kernel.org/

26. Creating a virtual build environment. http://people.canonical.com/ ogra/ arm/qemu/kernel/

27. BusyBox. http://www.busybox.net/

28. TY Ho, LB Chen, IJ Huang, An efficient HW/SW integrated verification methodology for 3D Graphics SoC development. in Proc of IEEE International Symposium of Consumer Electronics(ISCE2008). (2009)

29. LB Chen, CT Yeh, HY Chen, IJ Huang, A System-Level Model of Design Space Exploration for a Tile-Based 3D Graphics SoC Refinement, IEICE Transactions on Fundamentals of Electronics. Communications and Computer Sciences E92-A. 12, 3193-3202 (2009)

30. I Antochi, B Juurlink, S Vassiliadis, P Liuha, Memory Bandwidth Requirements of Tile-Based Rendering. in Proc SAMOS 2004, LNCS 3133. 323-332 (2004)

31. WH Chen, C Smith, S Fralick, A fast computational algorithm for the discrete cosine transform, Communications. IEEE Transactions on. 25(9), 1004-1009 (1977). doi:10.1109/TCOM.1977.1093941
32. WB Pennebaker, JL Mitchell, JPEG: Still Image Data Compression Standard. (Springer, 1993)

33. VirtualBox - a general-purpose full virtualizer. http://www.virtualbox.org/

doi:10.1186/1687-6180-2011-25

Cite this article as: Yeh et al:: Internet-based hardware/software co-

design framework for embedded 3D graphics applications. EURASIP Journal on Advances in Signal Processing 2011 2011:25.

\section{Submit your manuscript to a SpringerOpen ${ }^{\circ}$ journal and benefit from:}

- Convenient online submission

- Rigorous peer review

- Immediate publication on acceptance

- Open access: articles freely available online

- High visibility within the field

- Retaining the copyright to your article

Submit your next manuscript at $>$ springeropen.com 